\title{
Tecnura
}

\section{Análisis del comportamiento de los frenos de disco de los vehículos a partir de la aceleración del proceso de corrosión}

\section{Behavioral analysis of disc brake vehicle from accelerating the process of corrosion}

\author{
Ricardo Andrés García León*, María Angélica Acosta Pérez**, Eder Flórez Solano ***
}

Fecha de recepción: 23 de agosto de 2014

Fecha de aceptación: 6 de abril de 2015

Como citar: García León, R. A., Acosta Pérez, M. A., \& Flórez Solano, E. (2015). Análisis del comportamiento de los frenos de disco de los vehículos a partir de la aceleración del proceso de corrosión. Revista Tecnura, 19(45), 53-63. doi: 10.14483/udistrital.jour.tecnura.2015.3.a04

\section{RESUMEN}

El sistema de frenado de un automóvil debe satisfacer un complejo conjunto de requerimientos, entre los cuales la seguridad es lo más importante. Los frenos deben trabajar en forma segura y predecible en cualquier circunstancia, lo cual implica disponer de un nivel estable de fricción, en cualquier condición de temperatura, humedad y salinidad del medio ambiente.

Para un correcto diseño y operación de los discos de freno, es necesario considerar diferentes aspectos, tales como la geometría, el tipo de material, la resistencia mecánica, la temperatura máxima, la deformación térmica, la resistencia al agrietamiento, entre otros. Todos estos aspectos inciden en que el proceso de corrosión se acelere y se genere así desgaste de los componentes de los discos y por ende la inseguridad en el proceso de frenado.

Palabras clave: corrosión, deformación, desgaste, fricción, resistencia, seguridad, temperatura.

\begin{abstract}
The braking system of a car must meet a complex set of requirements, among which safety is paramount. The brakes must work safely and predictably under all circumstances, which means having a stable level of friction, under all conditions of temperature, as well as environmental factors.

For proper design and operation of the brake discs it is necessary to consider different aspects such as geometry, material type, mechanical strength, maximum temperature, thermal deformation, resistance to cracking, among others. All these aspects make the corrosion process be accelerated, generating component wear thus, and of course uncertainty in the braking process.
\end{abstract}

Keywords: corrosion, friction, resistance, safety, strain, temperature, wear.

* Ingeniero mecánico, estudiante de maestría en Ingeniería Industrial, profesional universitario. Universidad Francisco de Paula Santander Ocaña, Ocaña, Colombia. Contacto: ragarcial@ufpso.edu.co

** Ingeniera mecánica, magíster en Ingeniería Mecánica, Extensión Universitaria. Universidad Francisco de Paula Santander Ocaña, Ocaña, Colombia. Contacto: maacostap@ufpso.edu.co

*** Ingeniero mecánico, magíster en Ingeniería Mecánica, decano de la Facultad de Ingenierías. Universidad Francisco de Paula Santander Ocaña, Ocaña, Colombia. Contacto: enflorezs@ufpso.edu.co 


\section{INTRODUCCIÓN}

El sistema de frenos es, sin duda, el componente más importante para la seguridad vial del automóvil, ya que de este depende la detención total o parcial del vehículo, y en consecuencia la integridad de sus pasajeros. Generalmente el $70 \%$ de la energía cinética producida en el movimiento es absorbida por los frenos de disco delanteros y el restante por el freno trasero, que suele ser de tambor. Estos sistemas se fundamentan en la fricción para detener el movimiento del vehículo, teniendo como principio la presión hidráulica que empuja las pastillas de freno contra el disco de hierro fundido. En consecuencia, el comportamiento que produce este tipo de dispositivos, a través de la energía cinética, es crear un calor considerablemente alto durante el frenado, aumentando la temperatura por fricción; este calor se disipa rápidamente con el aire circundante por medio del fenómeno de convección (transferencia de calor que se produce entre masas a diferente temperatura). Los factores ambientales también son determinantes para que la etapa de transferencia de calor se produzca, y debido a estos comportamientos el proceso de corrosión se acelera rápidamente. Además, cuando la temperatura alcanza valores elevados aparece el fenómeno por radiación, que también ayuda a disipar la energía en forma de calor almacenada en el disco.
En estudios recientes, se presentó un modelo explicativo de la situación del contacto entre un complejo de pastillas de freno orgánico y un disco de hierro fundido. En este modelo, las fibras metálicas $u$ otros materiales duros en la almohadilla del material forman mesetas primarias estables o parches, que llevan a la parte principal de la carga (figura 1) (Wahlstróm, 2011):

De esto se pudo concluir que la dureza en las mesetas primaria y secundaria es aproximadamente la misma, y que la dureza del material de la matriz metálica es de aproximadamente 20 veces menor. Es decir, las mesetas más difíciles de proteger en el material de la matriz metálica se desgastan. En mediciones realizadas se encontró que las mesetas contacto normalmente pueden exhibir valores de dureza considerablemente más altos que la media dureza de la almohadilla compuesta (por ejemplo, en comparación $3.000 \mathrm{MPa}$ con $200 \mathrm{MPa}$ ), esto se debe a que las mesetas están típicamente formadas por fibras de metal rodeadas por los constituyentes de la matriz metálica más suaves y compactados (Söderberg \& Andersson, 2009).

Asimismo, con la disponibilidad actual de las herramientas de cálculo asistido por computador, tales como softwares comerciales para análisis mecánicos y térmicos, la simulación numérica es el método más adecuado y conveniente para analizar el comportamiento de los frenos de disco, ya sea durante su funcionamiento o bajo distintas

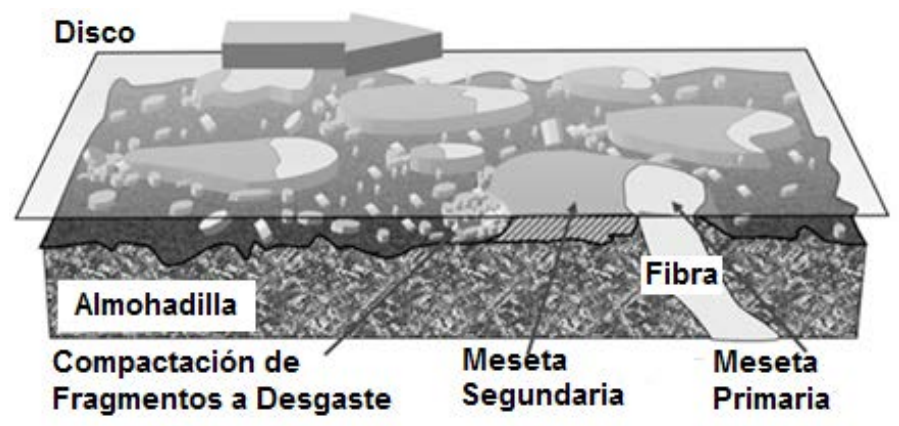

Figura 1. Ejemplo de la situación de contacto entre la pastilla y el disco.

Fuente: elaboración propia. 
condiciones de trabajo; sin embargo, en cualquier nuevo diseño se pueden emplear las herramientas de cálculo asistido por computador, las cuales deben ser validadas o comprobadas con ensayos de laboratorios y cálculos matemáticos. Estos métodos de estudio, análisis numérico y experimental deben estar incorporados en cualquier metodología de diseño de frenos de disco, si se desea que esta sea realmente válida.

\section{METODOLOGÍA}

Con el fin de tener una visión más amplia del comportamiento de los frenos de disco, se seleccionaron tres (3) tipos de muestras de diferentes vehículos, de los cuales se escogieron modelos de aquellos que se encuentran sometidos a distintas capacidades de carga o de transporte: un automóvil particular de la Renault, una camioneta de la Toyota y un microbus de transporte urbano de la Mitsubishi. Se realizó el análisis físico-mecánico del material de fabricación de los discos de cada uno de ellos y sus propiedades térmicas (calor específico, conductividad térmica, coeficiente de dilatación térmica, entre otras), para poder compararlos con aquellos encontrados en la literatura. Se realizaron ensayos de microestructura del material, características estructurales (tracción, compresión y dureza), además de los análisis térmicos y estáticos de los discos, en donde se simuló la geometría y los efectos de su comportamiento con el apoyo de los softwares ANSYS y SolidWorks.

Debido a la alta conductividad térmica, la buena resistencia al desgaste, la facilidad de fabricación y relativamente su bajo costo, el disco de freno es fabricado generalmente de una fundición gris, desde las primeras etapas de desarrollo del vehículo, pero a pesar de estas propiedades favorables, el comportamiento de la corrosión de hierro fundido sigue siendo un problema ya que a menudo contribuye a una situación indeseable llamada judder del freno, es decir, la aparición de manchas (Kikuchi, Hara, Sakairi, Yonezawa, Yonezawa, \& Takahashi, 2010).
Pocos estudios han examinado el tamaño, la forma o composición elemental con que los discos de freno se corroen tan rápidamente, fenómeno que depende de las condiciones a las cuales están enfrentados. Por tal motivo, se han desarrollado investigaciones sobre cómo la fricción genera fallas de corrosión y desgaste en los frenos, además del efecto en las propiedades mecánicas del material por las altas temperaturas que se producen (Ashby \& Jones, 2000).

Asimismo, se realizaron varios proyectos para investigar una alternativa, cómo materiales más ligeros podrían mejorar la eficacia del frenado al tiempo que contribuye a la eficiencia energética. Estos materiales de compuestos cerámicos incluidos y los materiales de carbono y grafito han encontrado un uso limitado (Blau, 2010).

Para implementar un nuevo material en la fabricación de discos de frenos, el coeficiente de fricción debe ser relativamente alto, pero lo más importante es que sea estable, independientemente de la temperatura, la humedad, el tiempo de uso, el grado de desgaste, la corrosión, la presencia de polvo y chorros de agua desde la carretera, etc. Además de estas propiedades, se deben tener en cuenta los requisitos para una larga vida y un alto confort, es decir, ausencia de vibración, ruido y chirrido (Blau, Jolly, Peter, \& Blue, 2007).

Durante frenadas normales, relativamente suaves, la fuerza de presión de la almohadilla contra el disco es de aproximadamente 5 KiloNewton (KN), lo cual resulta en un valor nominal de presión en la superficie de la almohadilla justo por encima de 1,2 MPa. En extremas situaciones, la presión podría ser aproximadamente de $10 \mathrm{MPa}$. Durante frenadas bruscas, la disipación de potencia en una pastilla de freno fácilmente excede de $30 \mathrm{~kW}$. Estas altas densidades de energía dan como resultado muy altas temperaturas de la superficie y por lo tanto ponen exigencias especiales en los materiales de fricción (Eriksson, Bergman, \& Jacobson, 2002).

Todas estas observaciones son parte de un proyecto sobre la generación del chirrido de los frenos. Como el chirrido por lo general se produce a bajas 
velocidades y a presiones moderadas, el freno se ha diseñado con un equipo de prueba destinado principalmente para esta aplicación. Esto significa que los frenos solo han estado expuestos al frenado en condiciones de velocidades relativamente bajas (0-25 km/h); estas pruebas son realizadas en el laboratorio con la humedad ambiente y temperatura casi constante $\left(20-25^{\circ} \mathrm{C}\right)$. En el transporte de mayores cargas en los vehículos se verán influenciados los componentes resistentes al desgaste en el coeficiente de fricción que se genera por su fracción de volumen. Por lo tanto, a pesar de los materiales que componen la estructura del disco de freno y de la pastilla se origina un desgaste en la mayor parte del volumen del revestimiento, esta situación se produce por el contacto metal - metal que existe entre los dos componentes (Jacobson, 2007).

Las altas temperaturas en la superficie del disco de freno, junto con el contacto deslizante directo de la guarnición de freno con el rotor, son responsables de las reacciones mecánicas y químicas, en este caso las reacciones triboquímicas que se producen durante el frenado. Debido a la compleja composición de las pastillas de freno, es difícil describir todas las reacciones triboquímicas en detalle. Un fenómeno típico que ocurre a menores temperaturas es una degradación de la resina fenólica. Otras reacciones reportadas como triboquímicas comprenden la oxidación de los componentes metálicos, por ejemplo hierro o cobre (Matějka \& Vlastimil, 2011).

Respecto a estudios de análisis numérico, se tomó como referencia un número considerable de discos de freno de los vehículos de extinción de incendios utilizados en el apoyo a las actividades aeronáuticas, a los cuales le fueron encontrados grietas mediante softwares especializados. La macrofractografía de los discos fabricados en hierro de fundición gris reveló que tenían varias grietas a lo largo del radio de las superficies de la pista del disco con una longitud aproximadamente en el rango de 1-7,5 cm. Además, mediante un análisis en microscopio óptico y de emisión de campo en un microscopio electrónico de barrido (MEB) se encontró que las grietas se propagaron en forma semielíptica a través del espesor de las superficies de fricción de acuerdo con un mecanismo de fatiga térmica. También se observaron zonas que mostraron signos definidos de calefacción por el efecto judder. El análisis de elementos finitos (FEA) se llevó a cabo para determinar el perfil de temperaturas en el disco, así como para estimar la distribución de tensiones durante el frenado. También se confirmó que las temperaturas relativamente altas alcanzadas durante las acciones de frenado, se atribuyen a la tensión de tracción residual que son ocasionadas por la repetición de la tensión térmica en ciclos, produciendo la aparición de las grietas en la superficie de contacto del disco (Bulthé \& Lise, 2008).

El análisis de elementos finitos (FEA) sirve para determinar el perfil de temperatura y además estimar el Von Mises. Hay resultados que muestran que las grietas son producidas por la fatiga térmica durante las etapas de calentamiento y enfriamiento de las acciones de frenado. En particular, debido al gradiente térmico producido a través del grosor del rotor; la tensión de tracción residual se produce durante el enfriamiento que se genera en el proceso de transferencia de calor por conducción. Posteriormente el FEA ha demostrado que tal tensión se localiza principalmente en la dirección radial de la superficie de fricción en el borde de los orificios de refrigeración (Bagnoli, Dolce, \& Bernabei, 2009).

\section{RESULTADOS Y DISCUSIÓN}

En esta sección se presentan los resultados obtenidos mediante los cálculos realizados a los parámetros críticos, en la medida en que estos son indispensables para la construcción y el buen funcionamiento de los frenos. Para tener un análisis claro mediante cálculos matemáticos es necesario conocer datos técnicos del vehículo, tales como: la masa del vehículo, distribución de pesos por eje, velocidad promedio, radio dinámico de la rueda, etc. De modo que los resultados numéricos puedan ser comparados con los obtenidos experimentalmente. 
Los cálculos que se tienen en cuenta en el desarrollo del proyecto se basan en el análisis de transferencia de calor por convección y máquinas hidráulicas debido a los ductos de ventilación presentes en la geometría.

Como se mencionó anteriormente, se tuvo en cuenta la geometría de tres diferentes tipos de frenos de disco con los datos iniciales mostrados en la tabla 1.

Tabla 1: Datos iniciales para la realización de los cálculos

\begin{tabular}{lccc}
\hline \multicolumn{1}{c}{ DISCO } & D.1 & D.2 & D.3 \\
\hline Masa del vehículo $(\mathrm{Kg})$ & 1250 & 1950 & 2250 \\
\hline Masa del disco $(\mathrm{Kg})$ & 3,80 & 4,25 & 6,10 \\
\hline
\end{tabular}

Fuente: elaboración propia.

Asimismo, a partir de estos datos se calcularon variables como la fuerza de frenado, la resistencia a la rodadura, el coeficiente de adherencia a la calzada, la fuerza de frenado en el pedal, el tiempo de frenado, la deceleración de frenado, la velocidad periférica, la energía disipada por el disco, la potencia disipada por el sistema de frenos, todo esto con el fin de calcular las temperaturas de contacto en las superficies de contacto de los discos, tomando como referencia el Enfriamiento de Newton, que es un concepto de transferencia de calor, el cual especifica que un objeto sometido a una temperatura $\mathrm{T}_{1}$, se enfriará si se deja a temperatura ambiente $\mathrm{T}_{\infty}$ de acuerdo con la ecuación (1) exponencial (Kim \& Dae, 2008).

$$
T_{i}-T_{\infty}=\left(T_{i}-T_{\infty}\right) * e^{-\frac{A * U}{m * C p} * t}
$$

Donde,

$T_{i}=$ Temperatura instantánea $\left({ }^{\circ} \mathrm{C}\right)$.

$T_{\infty}=$ Temperatura ambiente $\left({ }^{\circ} \mathrm{C}\right)$.

$\mathrm{A}=$ Área de transferencia de calor $\left(\mathrm{m}^{2}\right)$.

$U=$ Coeficiente de transmisión térmica.

$\mathrm{m}=$ Masa del disco de freno $(\mathrm{Kg})$.

$C_{P}=$ Calor específico del material $\left(\mathrm{J} / \mathrm{Kg}^{* \circ} \mathrm{C}\right)$.

$\mathrm{t}=$ Tiempo de enfriamiento de Newton $(\mathrm{s})$.
Se determinó que los discos de freno, luego de haber sido sometidos según los cálculos a unas temperaturas de 82,107 y $90{ }^{\circ} \mathrm{C}$, respectivamente, en una frenada, tomarán una temperatura ambiente de $20^{\circ} \mathrm{C}$ entre 40 a 60 minutos, siempre y cuando el enfriamiento se dé por convección natural, es decir, que el aire en el ambiente debe tener una velocidad igual a cero.

También se calculó la velocidad de pérdida de calor total frontal del disco, a partir de los diferentes tipos de convección que afectan al disco, como son: periférica y en la campana, mediante la siguiente ecuación (2).

$$
Q_{\text {Total }}=Q_{\text {Periférica }}+Q_{\text {Campana }}
$$

De la cual se sabe que en la ecuación (3):

$$
Q=h * A_{s} *\left(T_{s}-T_{\infty}\right)
$$

Donde,

$\dot{Q}=$ Velocidad de pérdida de calor $(\mathrm{W})$.

$\mathrm{h}=$ Coeficiente de convección $\left(\mathrm{W} / \mathrm{m}^{2 * \circ} \mathrm{C}\right)$.

$A_{S}=$ Área superficial del disco de freno $(\mathrm{m} 2)$.

$T_{S}=$ Temperatura superficial $\left({ }^{\circ} \mathrm{C}\right)$.

$T_{\infty}=$ Temperatura ambiente $\left({ }^{\circ} \mathrm{C}\right)$.

Además se tuvieron en cuenta los diferentes tipos de convección que están presentes en el disco, como la convección lateral, la producida por el efecto rotatorio y la generada en el interior de los ductos de ventilación.

Para nuestros propósitos de análisis numérico de los discos de freno, no existe un software que realice las resoluciones conjuntas de los problemas físico-mecánicos y termo-mecánicos no lineales (cálculos acoplados). Esto hizo que se plantearan varias fases en la metodología propuesta y se utilizaran los Software ANSYS y SolidWorks para distintas disciplinas, y de esta manera encontrar los resultados analíticos del modelo termo-mecánico por FEM.

Durante la puesta a punto de la metodología, la comparación de resultados entre la simulación y la experimentación de los ensayos de laboratorio 
fue un objetivo importante. Es fundamental contar con los equipos adecuados para medir las variables y así poder definirlas en los análisis numéricos (Criado, 2012).

Para comprobar y analizar los resultados se realizó la correcta geometría de los tres discos de freno, que fueron diseñados en el software SolidWorks, para efectos de la ejecución de la simulación (figuras 2, 3 y 4).

En el caso de las propiedades estructurales, el esfuerzo de tensión es mínimo, como por ejemplo en la compresión: la resistencia a compresión de las fundiciones grises es alta: 3 o 4 veces superior a la resistencia a la tracción. Este comportamiento se debe a que, en compresión, apenas influye el efecto de entalla del grafito y, por tanto, la contribución negativa de este se reduce simplemente a disminuir la sección eficaz del constituyente matriz metálica.

Por tal razón fue necesario calcular el esfuerzo de tensión, los límites elásticos y la resistencia de los materiales a la plasto-deformación y la fatiga, que son extremadamente importantes en ingeniería, ademas del límite de elasticidad, el límite de fluencia y el límite de resistencia.

En el caso de las probetas de compresión, los extremos deben ser planos para que no causen concentraciones de esfuerzos, y deben ser perpendiculares al eje de la pieza para que no causen flexión debida a carga excéntrica, como se muestra en las figuras 5 y 6 .

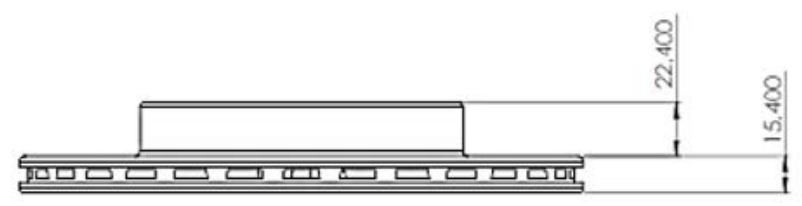

Figura 2. Muestra del disco de freno 1 para el análisis de los resultados.

Fuente: elaboración propia.

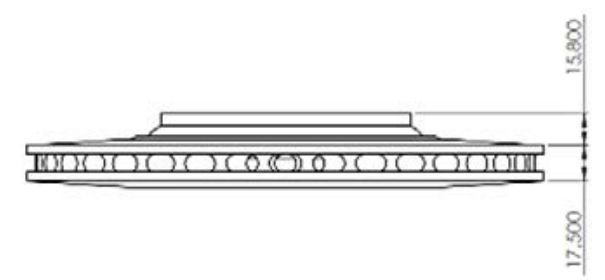

Figura 3. Muestra del disco de freno 2 para el análisis de los resultados.

Fuente: elaboración propia.

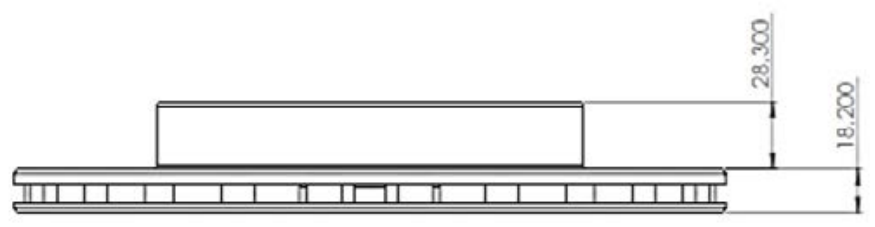

Figura 4. Muestra del disco de freno 3 para el análisis de los resultados.

Fuente: elaboración propia. 


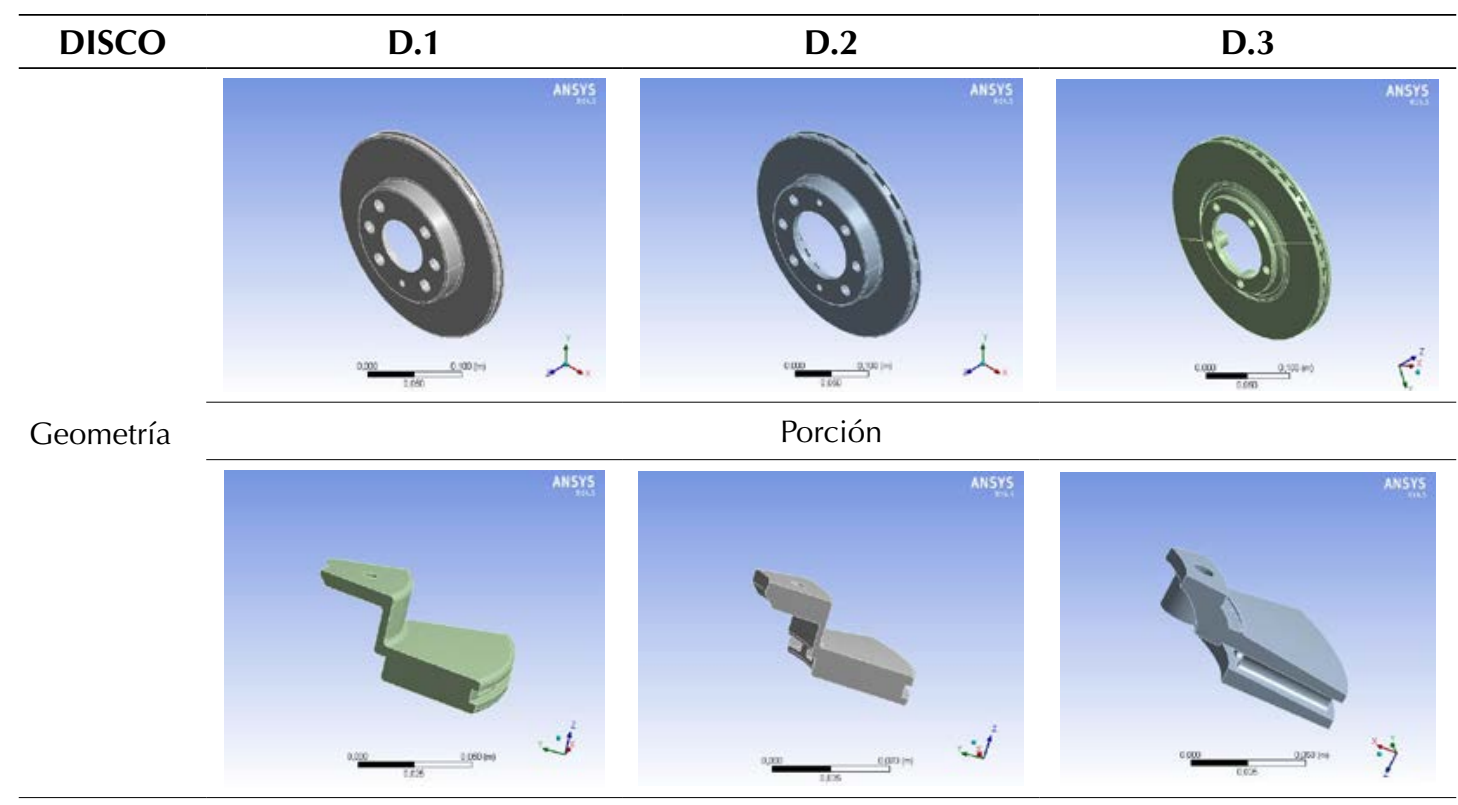

Figura 5. Geometría de los discos realizados en el software SolidWorks.

Fuente: elaboración propia.

El ensayo de compresión se realizó con esta geometría debido a que la norma ASTM E9 especifica el tamaño mínimo para las probetas.

En la figura 7 se puede observar que las deformaciones más grandes se presentan en los extremos de los discos y que son milimétricas a causa del desgaste generado por el área de contacto con las pastillas del sistema de frenado, por lo cual se producen altas temperaturas, desgaste y aceleración del proceso de corrosión.

Asimismo, también se realizó el ensayo de dureza para determinar qué características de maquinabilidad tenía el material y de esta forma comprobar y evaluar las condiciones de compresión a las cuales es sometido este tipo de frenos. Se obtuvieron los siguientes resultados (tabla 2):

Tabla 2. Ensayo de dureza y sus resultados promedios

\begin{tabular}{lccc}
\hline \multicolumn{1}{c}{ DISCO } & D.1 & D.2 & D.3 \\
\hline Valor Promedio HRB & 84,7 & 80,2 & 82,0 \\
\hline Valor HB & 164 & 151 & 156 \\
\hline
\end{tabular}

Fuente: elaboración propia.
En el caso de la fundición gris para este tipo de frenos se determinó que poseen una dureza de entre 140 y 250 Brinell, lo cual significa, según la bibliografía, que este tipo de material puede mecanizar fácilmente.

El análisis metalográfico fue realizado con el objetivo de obtener las características principales de las fundiciones grises, en lo que respecta a la microestructura y morfología del material, ya sea la de los grafitos o de las fases presentes (ferrita y perlita), y de los grafitos, ya sea el tamaño, el tipo, las fases o su distribución, como se pueden observar en la figura 8.

Por otra parte, la caracterización de los elementos presentes en la composición química de este tipo de frenos de disco tuvo el objetivo de conocer el efecto de los elementos de la aleación de manganeso, silicio, cromo y demás componentes de la matriz metálica que influyen en la microestructura del material (Sanz Pero, 2000).

Asimismo, se utilizó la bibliografía especificada para las propiedades estructurales de los hierros de fundición gris y se comparó con los resultados 


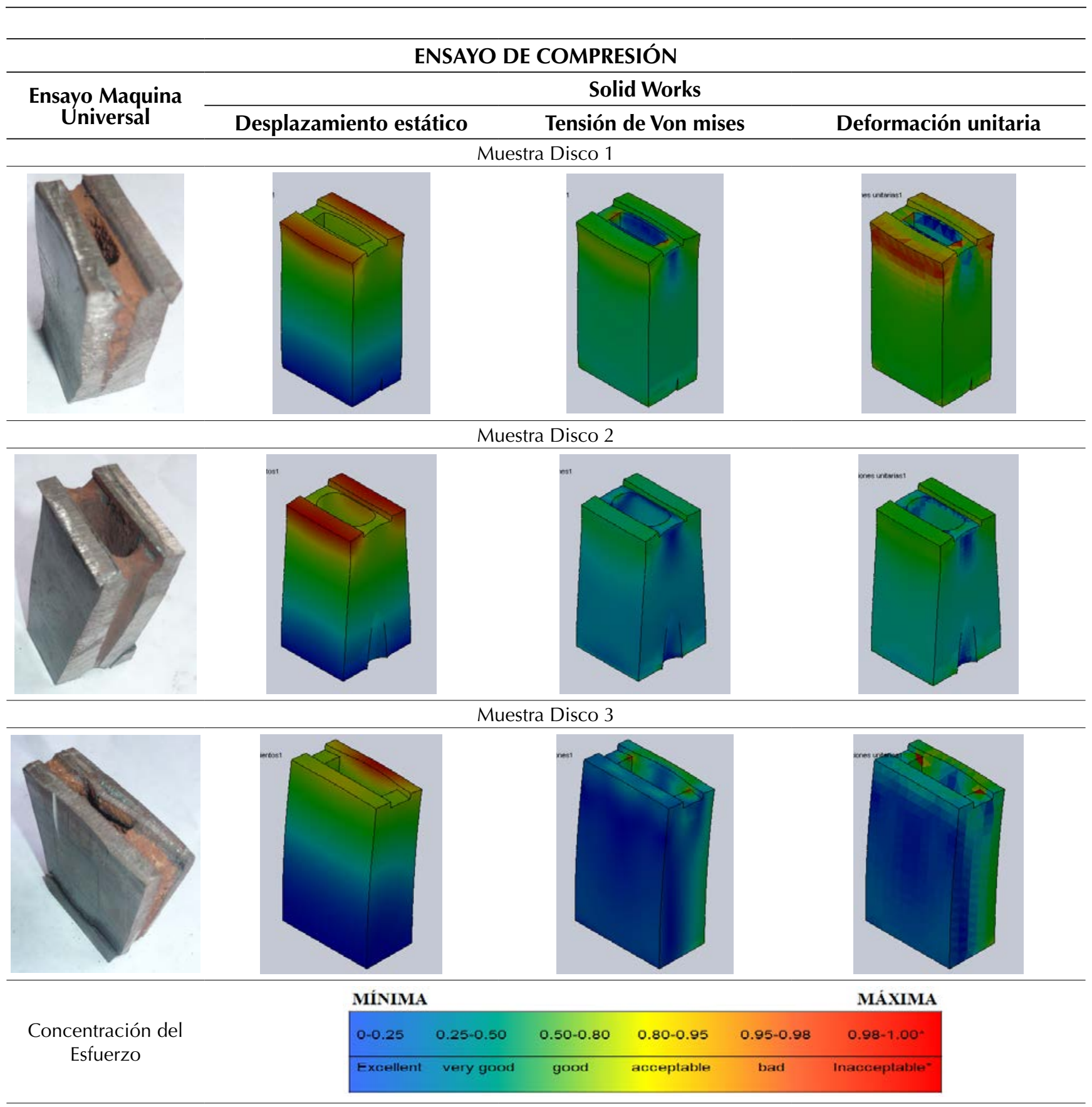

Figura 6. Ensayo de compresión y sus resultados analizados en el software SolidWorks.

Fuente: elaboración propia.

obtenidos en el microscopio, en donde las imágenes obtenidas sí coincidían con las características físicas de una fundición gris.

Finalmente se realizó el análisis termo-mecánico con el propósito de predecir las temperaturas, los correspondientes esfuerzos y deformaciones de origen térmico en el disco de freno. Como resultado de este análisis se encontró que el calor generado por fricción entre las pastillas del freno y el disco se distribuye uniformemente sobre todo el área exterior de las pistas del freno de disco, por lo anterior el flujo de entrada de calor $Q$, se calcula a partir de la 
velocidad de rotación y de la fuerza de fricción, para unas condiciones particulares de funcionamiento.

Además, como para este análisis térmico es necesario tomar un punto de partida, determinamos la energía y potencia disipada en el proceso de frenados como primer paso, calculados con las ecuaciones (1), (2) y (3).
Mediante la realización del análisis térmico a los tres discos de frenos se pudo observar con una visión más amplia el comportamiento de la distribución de la temperatura, el flujo de calor y la dirección del flujo de los discos en toda su geometría, lo que es de gran interés para la investigación.

DISCO D.1 D.2 D.3

Figura 7. Deformaciones totales analizadas en el software ANSYS.

Fuente: elaboración propia.

\begin{tabular}{ccc}
\hline & Fundición gris & \\
\hline Acercado a 50X & Acercado a 400X & Acercado a 600X \\
\hline & &
\end{tabular}

Figura 8. Imágenes de las muestras en el microscopio.

Fuente: elaboración propia 


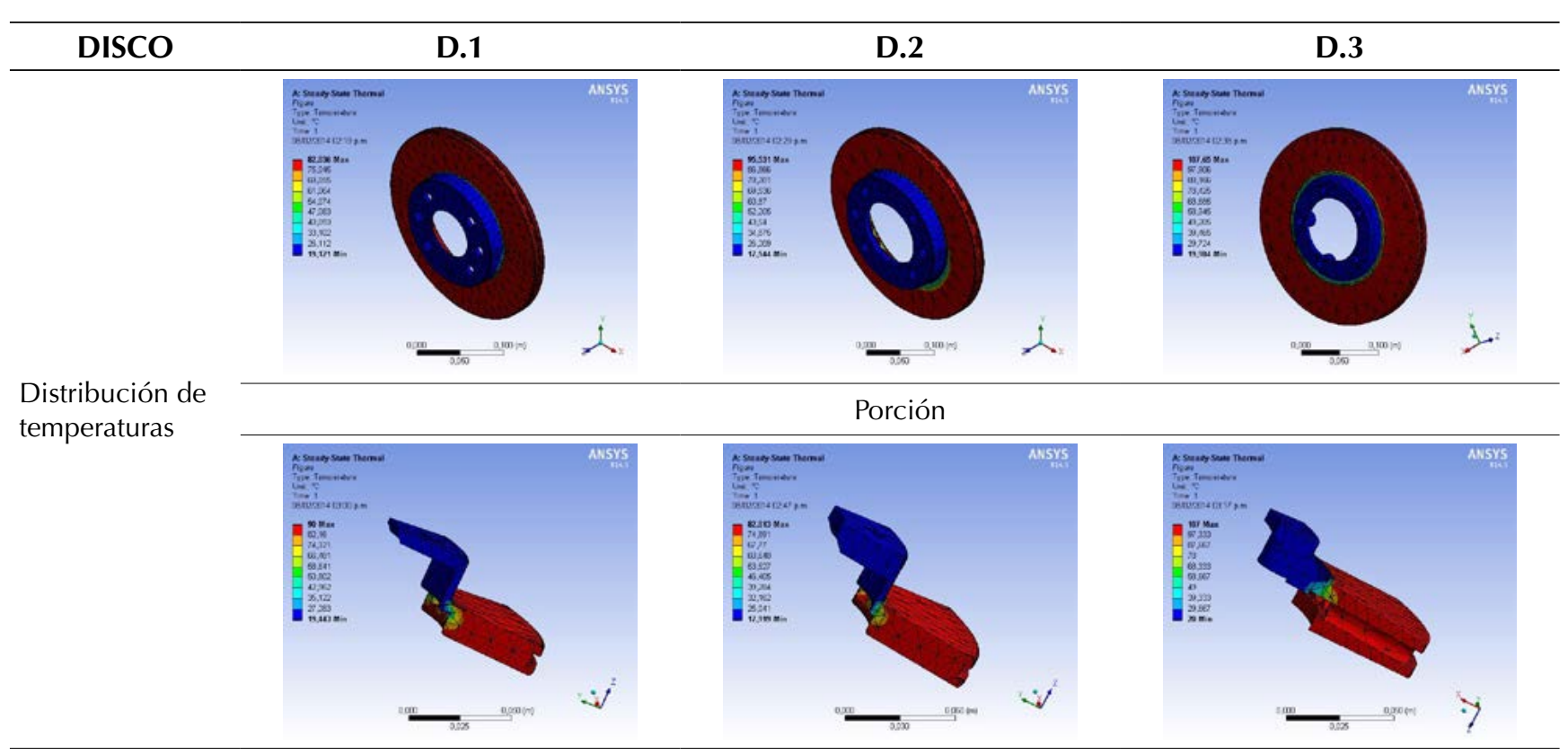

Figura 9. Distribución de temperaturas y los resultados analizados en el software ANSYS.

Fuente: elaboración propia.

Teniendo en cuenta las condiciones iniciales de funcionamiento que fueron calculadas, y mediante programas de elementos finitos, se pudieron observar las zonas de influencia por la distribución de temperaturas generadas entre la pastilla y el disco del freno. Este comportamiento se presenta en la figura 9, en la cual se observa la mayor temperatura alcanzada en cada uno de los discos ventilados.

\section{CONCLUSIONES Y RECOMENDACIONES}

El sistema que los autores de la investigación consideraron motivo de estudio y cálculo fue el más adecuado, debido a que los elementos en consideración son de fácil acceso y hoy en día casi la totalidad de los vehículos poseen frenos de disco ventilados en las cuatro ruedas por su efectividad en el proceso de frenado; caso contrario ocurre en vehículos que continúan utilizando los frenos de tambor en el eje trasero, y por lo general la mayoría de los inconvenientes como la perdida de calor, humedad y de esta manera la corrosión que proceden de la ineficiencia del frenado, debido a que las zonas de generación de calor se encuentran cerradas se retrasa la evacuación de calor y por consiguiente se deterioran las propiedades estructurales del material. Así mismo, el uso excesivo de los frenos puede ocasionar la ebullición del líquido de frenos y con esta la pérdida parcial o total del sistema de frenado. (Piña, 2008)

Gracias a las imágenes obtenidas en el microscopio se comprobó la microestructura y caracterización de las láminas de grafito que están presentes en los tres discos fabricados de este tipo de fundición gris, resultados que fueron comparados y analizados con la bibliografía. Asimismo, con la simulación en el software SolidWorks y la realización del ensayo de compresión en la máquina universal, se pudieron observar los fenómenos estáticos tales como: esfuerzos, deformaciones y el factor de seguridad, a los cuales están sometidos los frenos de disco de los vehículos, además del deterioro y pérdida en sus propiedades estructurales, las cuales son afectadas por la influencia de la aceleración de la corrosión (Roadhause, 2006). 
La aceleración del proceso de corrosión en los frenos de disco es influido específicamente por los cambios térmicos a los cuales está expuesto el disco por la acción de la fricción entre las pastillas, la interacción con el medio al cual se encuentra expuesto, la utilización de un líquido para frenos con características inadecuadas, además de la falta de mantenimiento de los componentes del sistema de frenado, lo cual conduce a que las propiedades físico-mecánicas y estructurales del disco resulten afectadas.

\section{FINANCIACIÓN}

El proyecto se desarrolló con recursos propios, con un estimado aproximado de $\$ 845.000$ pesos moneda corriente, en lo que concierne a gastos en préstamo de equipos de laboratorios y compra de materiales, además de otros gastos derivados del desarrollo del proyecto.

\section{REFERENCIAS}

Ashby, M., \& Jones, D. (2000). Engineering Materials. Boston: Heinemann.

Bagnoli, F., Dolce, F., \& Bernabei, M. (2009). Thermal fatigue cracks of the fighting vehicles gray iron brake discs. Engineering Failure Analysis , 16 (1), 152-163.

Blau, P. (2010). Compositions, funtions, and testing of friction brake materials and their additives. Oak Ridge National Laboratory Tecnical Report ORNL/ $T M, 64,24-27$.

Blau, P., Jolly, B., Peter, W., \& Blue, C. (2007). Tribological investigation of titanium-based materials for brakes. Science, 263 (7), 1202-1211.

Bulthé, ,. C., \& Lise, A. (2008). Mechanical and chemical investigation of the temperature influence on the tribological mechanisms occurring in OMC/cast iron friction contact. Science, 264 (9), 815-825.
Criado, E. (2012). Diseño y cálculo del sistema de frenado para un prototipo formula student. Leganes, España: Universidad Carlos III de Madrid.

Eriksson, M., Bergman, F., \& Jacobson, S. (2002). On the natura of tribological contact in automotive brakes. Science, 252 (1), 26-36.

Jacobson, R. (2007). Applications of a new model for the abrasive wear resistance of multiphase materials. Composites and Coated Materials, 174, 1459-1463.

Kikuchi, T., Hara, Y., Sakairi, M., Yonezawa, T., Yonezawa, A., \& Takahashi, H. (2010). Corrosion of AlSn-Bi alloys in alcohol at high temperatures. Part I: Effects of the metallurgical structure of the alloys and the metal salt additions to alcohol. Corrosion Science, 52 (4), 1482-1491.

Kim, \& Dae, J. (2008). Thermal stress analysis for a disk brake of railway vehicles with consideration of the pressure distribution on a frictional surface. Materials Science and Engineering , 483, 456-459.

Matějka, \& Vlastimil. (2011). Possible stibnite transformation at the friction surface of the semi-metallic friction composites designed for car brake linings. Applied Surface Science , 258 (5), 1862-1868.

Piña, M. (2008). Estudio de la influencia de la morfología grafítica sobre las propiedades mecánicas de los discos de freno automotrices. México: Instituto Politécnico Nacional.

Roadhause. (2006). Manual técnico de la pastilla de freno (Discos de frenos). España: Road.

Sanz Pero, J. (2000). ACEROS. En Metalurgia Física Selección y diseño (págs. 159-161). Madrid, España: CIE Dossat.

Söderberg, A., \& Andersson, S. (2009). Simulation of wear and contact pressure distribution at the padto-rotor interface in a disc brake using general purpose finite element analysis software. Science Direct, 267 (12), 2243-2251.

Wahlstróm, J. (2011). A study of airborne wear particles from automotive disc brakes. The Royal Institute of Technology, 11, 1040-1179. 
